\title{
Reduction of Scan Time and Echo Time in 3D Time-of-Flight (TOF) MR Angiography, Using Reverse Elliptical Centric Dual- Echo Acquisition
}

\author{
Mingliang Chen ${ }^{1}$, Zhaoyang $\mathrm{Jin}^{1}$, Zhixing Wang ${ }^{4}$, and Yiping P. Du ${ }^{2,3}$, a \\ ${ }^{1}$ Department of Automation, Hangzhou Dianzi University \\ ${ }^{2}$ Department of Biomedical Engineering, Shanghai Jiao Tong University \\ ${ }^{3}$ Shanghai United Imaging Healthcare co.,Itd \\ ${ }^{4}$ Department of Biomedical Engineering, Zhejiang University
}

\begin{abstract}
In the conventional single-echo three dimension (3D) time-of-flight (TOF) magnetic resonance angiography (MRA), it always takes long scan time to obtain the good images. In addition, high-resolution MRA with flow compensation requires long echo time (TE). Here, a reverse elliptical centric dual-echo 3D TOF MRA pulse sequence was developed to reduce the scan time and minimize the effective TE. Dual-echo and reverse elliptical centric acquisition in the phase encoding and slice phase encoding direction were used to reduce the scan time by $61 \%$. Furthermore, the effective echo time (TE) was also minimized from $4.8 \mathrm{~ms}$ to $4.1 \mathrm{~ms}$. The developed pulse sequence was tested on clinical MRI systems and the contrast to noise ratio (CNR) was measured to evaluate the results. The results show that the CNR only decrease by $2.5 \%$ with the scan time reduced to $39 \%$ when compared with the conventional single-echo $3 \mathrm{D}$ TOF. Compared with the conventional dual-echo 3D TOF acquisition, the developed pulse sequence brings advantages in three aspects: (1) reduce scan time by $22 \%$; (2) increase CNR by $10 \%$; (3) reduce the artifacts induced by dual-echo effects. In addition, while keeping the similar scan time as the conventional single-echo 3D TOF acquisition, the results show that reverse elliptical centric dual-echo 3D TOF acquisition increase CNR by $54.3 \%$.
\end{abstract}

\section{Introduction}

In magnetic resonance imaging (MRI), the image degradation due to the signal loss remains a significant problem. And TOF MRA is sensitive to signal loss in two basic mechanisms: (1) saturation of flowing spins (2) intra-voxel spin phase dispersion. Here we mainly discuss about the second mechanis m. In TOF MRA, it can be caused by two factors: (1) the inhomogeneity of the main magnetic field, the influence of eddy current and magnetic susceptibility; (2) the complex flow of blood, such as acceleration flow, pulsation ${ }^{1}$. Previous studies have indicated that the signal loss caused by the intra-voxel spin phase dispersion is greatly improved in short echo time (TE). Thus, on the previous basis using partial RF puls $\mathrm{e}^{2}$, the maximum amplitude of the flow compensation gradients, as well as partial echo acquisition technology ${ }^{34}$ and so on, the novel design for 3D TOF in this article reduce the TE further to improve the blood vessel imaging.

In the conventional 3D TOF single-echo imaging, the scan time often requires more than 10 minutes due to the phase encoding in two directions and the use of multiple overlapped thin slab

\footnotetext{
${ }^{a}$ Corresponding author: yipingdu@sjtu.edu.cn
} 
(multi-slab) $)^{5}$. Therefore, it's difficult for the patients to tolerate it, which will absolutely affect the final imaging. To solve this, someone proposed the conventional dual-echo $3 \mathrm{D} \mathrm{TOF}^{6}$ to reduce the scan time by $50 \%$ when compared with the conventional single-echo $3 \mathrm{D}$ TOF. In this paper, a reverse elliptical centric dual-echo 3D TOF pulse sequence is developed, and the scan time can be decreased to $39 \%$ of the conventional single-echo $3 \mathrm{D}$ TOF.

\section{Principle and method}

\subsection{First order flow-compensation}

For the 3D TOF pulse sequence, the first order flow compensation is applied on the readout and slice phase encoding directions to null the first-order moment at the peak of the echo.

Figure 1 shows a first order flow-compensation diagram. $G_{\text {max }}$ and $T_{r}$ are the maximum gradient amplitude and the corresponding shortest ramp time, respectively. The widths of the flowcompensation gradient pairs along $k_{z}$ direction are derived as: ${ }^{78}$

$$
\begin{gathered}
W_{1}^{Z}=\frac{1}{2}\left\{-3 T_{r}+\sqrt{T_{r}^{2}+4\left[\frac{A_{Z}-M_{0}}{G_{\max }}\left(\beta+\frac{T_{r}}{2}+\frac{A_{Z}-M_{0}}{2 G_{\max }}\right)-\frac{M_{1}}{G_{\max }}\right]}\right\} \\
W_{2}^{Z}=\frac{A_{Z}-M_{0}}{G_{\max }}+\frac{1}{2}\left\{-3 T_{r} \pm \sqrt{T_{r}^{2}+4\left[\frac{A_{Z}-M_{0}}{G_{\max }}\left(\beta+\frac{T_{r}}{2}+\frac{A_{Z}-M_{0}}{2 G_{\max }}\right)-\frac{M_{1}}{G_{\max }}\right.}\right]
\end{gathered}
$$

where $A_{z}$ are the net encoding areas along the phase and slice directions, respectively, and they must be incremented with constant steps with the differences being between two consecutive steps proportional to $\Delta \mathrm{k}_{\mathrm{z}}$. $\beta$ is the time between the end of the second flow compensation pulse with $\mathrm{W}_{2}^{\mathrm{z}}$. $\mathrm{W}_{0}, \mathrm{~T}_{\mathrm{r}}^{0}$, and $\mathrm{G}_{0}$ are the widths of the plateau, the ramp time, and the amplitude of the plateau, respectively. $\mathrm{M}_{0}^{0}$ and $\mathrm{M}_{1}^{0}$ are the zeroth and first moments of the slab-selection gradient.

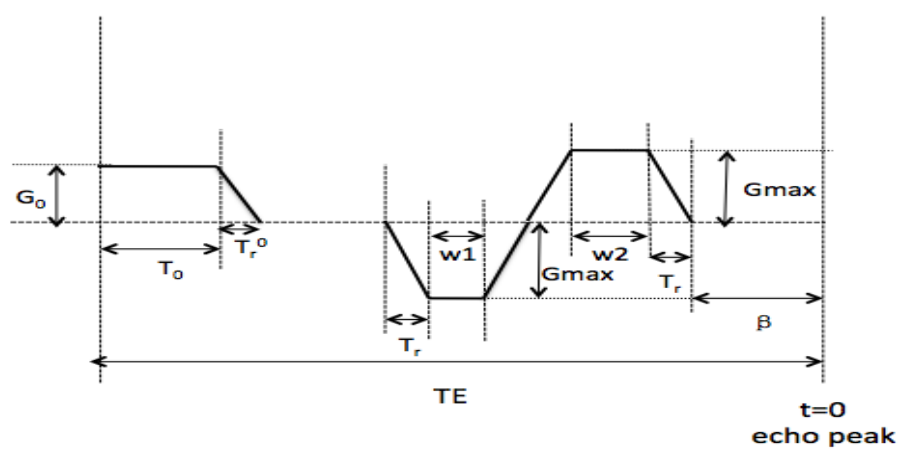

Figure 1. Gradient diagram of the flow-compensations along the slice phase-encoding directions for 3D TOF imaging. It starts from the center of RF pulse and ends in the echo peak.

\subsection{Revers elliptical centric dual-echo view order}

To realize this, each of the $\left(\mathrm{N}_{\mathrm{y}} \times \mathrm{N}_{\mathrm{z}}\right)$ views in the $\mathrm{k}_{\mathrm{y}}-\mathrm{k}_{\mathrm{z}}$ plane is initially sorted according to their distance from the center of $\mathrm{k}$-space. The views that are outside of the ellipse:

$$
\frac{k_{y}^{2}}{k_{y \max }^{2}}+\frac{k_{z}^{2}}{k_{z}^{2} m x}=1
$$


but inside the standard $\mathrm{k}_{\mathrm{y}}-\mathrm{k}_{\mathrm{z}}$ rectangle are skipped. As shown in the Fig. 2, the open dots in the four corners are the omitted views, which are not acquired and substituted with zeros during reconstruction. And in the previous work, it has been shown that the elliptical views can reduce the scan time of 3D acquisition by $21 \%$ with a slight penalty on the image quality ${ }^{9}$. Next, the dots located in the ellipse are further divided into two equal parts according to the distance fro $\mathrm{m}$ the orig in of $\mathrm{k}$-space. The red dots are the views close to the center of $\mathrm{k}$-space and are acquired in the first echo with a relatively short echo time (TE), determining the signal-noise ratio (SNR) and contrast-noise ratio (CNR) of the images. The black dots are the views far away from the origin of k-space and are acquired in the second echo with a long TE. For dual-echo, two echoes are acquired within one repetition time (TR). The SNR and CNR of images are mainly determined by the first echo, which is also called the effective TE. Starting from their respective edge, both red dots and black dots with the views of the decreasing distance from the orig in of k-space are acquired subsequently.

Such design has brought some advantages. (1) Starting from the periphery of k-space views, it can reduce the use of dummy scans (i.e., scan with the data acquisition disabled), which are used to reach a steady state ${ }^{8}$. (2) By collecting the views close to the center of $k$-space centrally, the artifacts can be reduced. Because 3D TOF acquisitions are commonly phase encoded in two orthogonal directions, there is considerable flexibility in the $\mathrm{k}_{\mathrm{y}}-\mathrm{k}_{\mathrm{z}}$ view order. Using the reverse elliptical centric view order, the conspicuous ghosts can be reduced by evenly spreading the ghosts in two dimensions (phase and slice $)^{10}$. (3) Combining the dual echo with reverse elliptical centric view order techniques, the scan time can be reduced to about $39 \%$ of the conventional single-echo $3 \mathrm{D}$ TOF. In addition, owing to the decrease of the slice phase encoding area in the first echo, from the calculation equations (1) and (2) in the flow compensation part above, the effective TE can be reduced. The degree of reduction is determined by the maximum gradient and corresponding ramp time used in the design and the number of phase encodings in the slice direction.

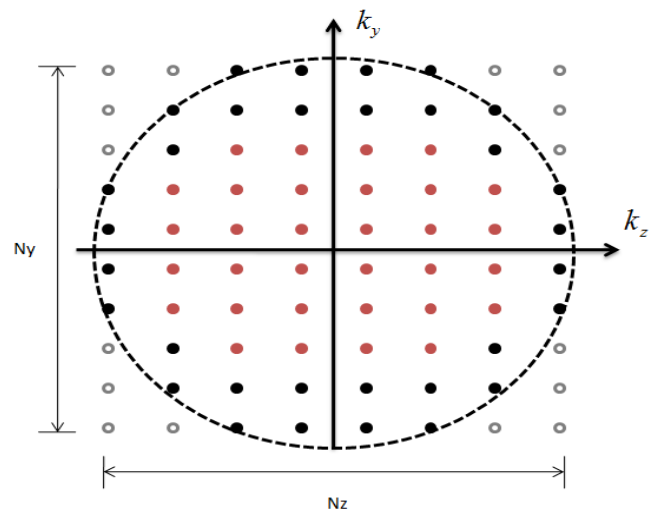

Figure 2. The views order in $k_{y}-k_{z}$ space for reverse elliptical centric dual-echo 3D TOF acquisition. The data for the views in the corners are not acquired and set to zeros during image reconstruction. The remaining views (the solid black dots and red dots) are further divided into two equal parts according to the distance from the origin of k-space. The solid red dots and the black dots are acquired in the first and second echo, respectively.

\subsection{MRI}

To demonstrate the potential advantages of reverse elliptical dual-echo 3D TOF acquisition, an initial comparis on was made with the conventional single-echo 3D TOF and the conventional dual-echo 3D TOF acquisition, respectively. 


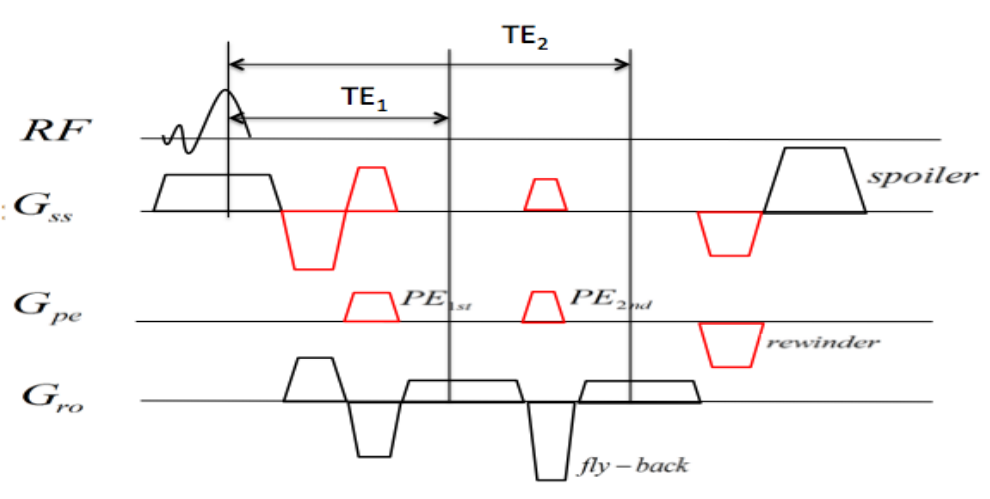

Figure 3. Diagram of the reverse elliptical centric dual-echo 3D TOF pulse sequence. To achieve the short TE, the asymmetric RF pulse, partial-echo acquisitions and flow-compensated gradients with minimum duration are applied in the sequence. In addition, by reducing the slice phase-encoding areas of the first echo, the effective TE $\left(T E_{1}\right)$ is shortened. A fly-back gradient is located in the middle of two echoes to restore flow compensation in the second echo. And the gradients in phase-encoding and slice phase-encoding directions are rearranged to accomplish the reverse elliptical centric dual-echo view order. The red gradient means that the gradients will vary according to the specific view order.

Figure 3 shows the reverse elliptical centric dual-echo 3D TOF sequence. The acquisition of a second echo with the extra phase encoding and slice encoding is added to a conventional multiple overlapped thin slab acquisition of a 3D TOF pulse sequence. Both the view orders of the two echoes are rearranged to cover the elliptical $\mathrm{k}_{\mathrm{y}}-\mathrm{k}_{\mathrm{z}}$ space.

Five healthy volunteers were scanned on a $1.5 \mathrm{~T}$ MR scanner (United Imaging Healthcare, Shanghai, China) using a 16-channel phased array head coil, the maximal gradient field adopted here was the $15.5 \mathrm{mT} / \mathrm{m}$, and the corresponding ramp time was $270 \mathrm{us}$. For the three pulse sequences, an asymmetric RF pulse and $71 \%$ of partial echoes acquisition were used to reduce TE. The effective TE of conventional single-echo 3D TOF sequence and conventional dual-echo 3D TOF were both limited to $4.8 \mathrm{~ms}$, but was reduced to $4.1 \mathrm{~ms}$ in the reverse elliptical centric dual-echo 3D TOF. In addition, the second TE in the conventional dual-echo 3D TOF and reverse elliptical centric dual-echo 3D TOF acquisition were $11.8 \mathrm{~ms}$ and $11.1 \mathrm{~ms}$, respectively. The other parameters for these scans were same, i.e., 22cm field-of-view (FOV), 256*256 matrix, $1 \mathrm{~mm}$ slice thickness, $25^{\circ}$ flip angle, $26 \mathrm{~ms}$ repetition time (TR), $\pm 30 \mathrm{kHZ}$ bandwidth (BW). To reduce the inflow saturation ${ }^{11}$, four slabs were acquired with 24 slices per slab and a $25 \%$ overlap between two slabs. In order to avoid the signal wrap-around along the slice-selection direction, the imaged slab width was set as $28 \mathrm{~mm}$ corresponding to $24 \mathrm{~mm}$ wide for the excited slab.

Images reconstruction and post-processing were performed by using MATLAB. In order to reduce the partial volume effect ${ }^{12}$, zero-filling interpolation ${ }^{13}$ was applied to k-space data to reconstruct 3D complex images with a total $256^{*} 256^{*} 156$ matrix. Then CNR was measured on the final MRA images, including different anatomic locations. To quantify the CNR, the method developed by Du et al and refined by Chapman et $\mathrm{al}^{14}$, was used.

\section{Results}

Figure 4 shows the projections of the MRA images acquired by the conventional single-echo 3D TOF acquisition in the left column and the reverse elliptical centric dual-echo 3D TOF acquisition in the right column, respectively. The three rows correspond to the MIP (maximum-intensity projection) of MRA in the transverse, sagittal and coronal plane. The CNR of the four vessels indicated by the arrows in the Fig. 4 was measured to evaluate the performance. To distinguish them, the four blood 
vessels were marked in four different colours, and they are red (vessel one), blue (vessel two), green (vessel three) and yellow (vessel four).
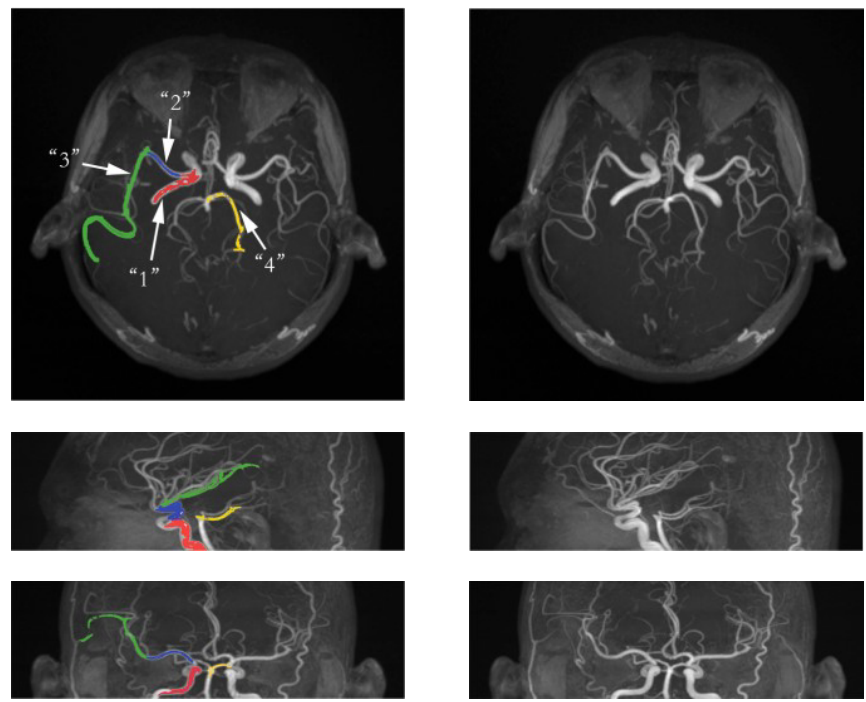

(a)

(b)

Figure 4. The MRA images acquired by reverse elliptical centric dual-echo 3D TOF (column a) and conventional single-echo 3D TOF (column b) acquisition. From top to bottom, the MIP of MRA in the transverse, sagittal, coronal plane were shown to observe the difference. After reducing the scan time by $61 \%$ and the effective TE about $0.7 \mathrm{~ms}$, the MRA image of reverse elliptical centric dual-echo 3D TOF method maintains the similar vascular visualization with the conventional single-echo 3D TOF method.

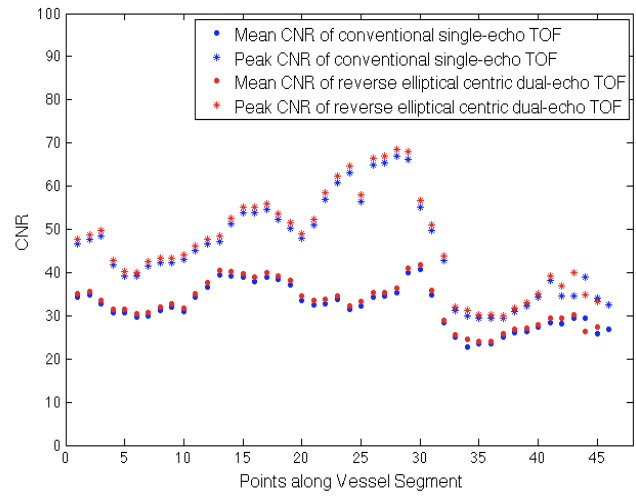

(a)

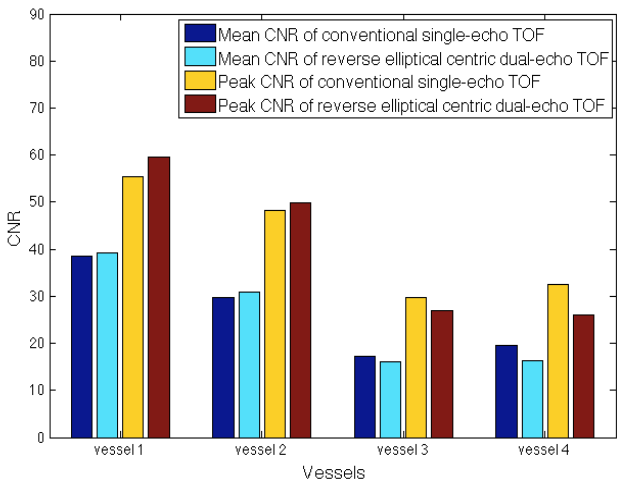

(b)

Figure 5. The mean CNR and peak CNR of the points along the big vessel labeled " 1 " in conventional singleecho and reverse elliptical centric dual-echo 3D TOF acquisition are shown in (a). A histogram shown in (b) is used to graphically summarize and display the mean CNR and p eak CNR of four vessel segments in Fig.4.

Fig.5 (a) shows the representative local vessel CNRs of the big vessel "1". The solid points designate the mean local vessel CNR and the asterisks designate the peak local ves sel CNR. The histogram in Fig.5 (b) shows the mean CNR and peak CNR of the four vessel segments averaged from five volunteers. From analys is of the results, it can be found that for the big vessels like vessel one, two, the CNR of reverse elliptical centric dual-echo 3D TOF MRA is a little higher than that in the conventional single-echo 3D TOF MRA. And the CNR for small vessels, such as vessel three, four, decrease a little because of the long echo time of the acquisitions for high frequency parts. 
Furthermore, the blurring and ghosting due to off-resonance effects that can be predicted theoretically by point spread function (PSF) analys is ${ }^{15}$ may also degrade the CNR.

In conclusion, compared with the conventional single-echo 3D TOF, only 39 percentage of the scan time is taken, but the MRA with similar CNRs is obtained in the reverse elliptical centric dualecho 3D TOF pulse sequence.

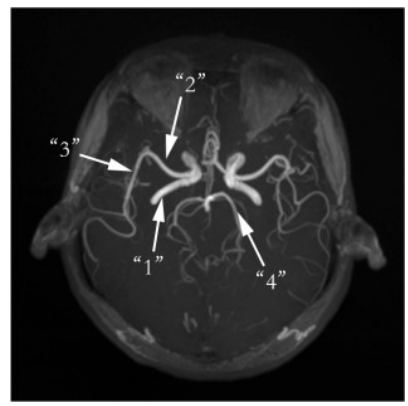

(a)

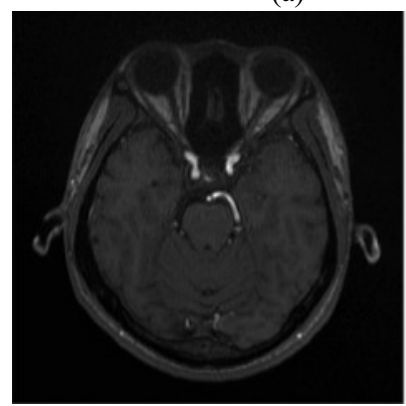

(c)

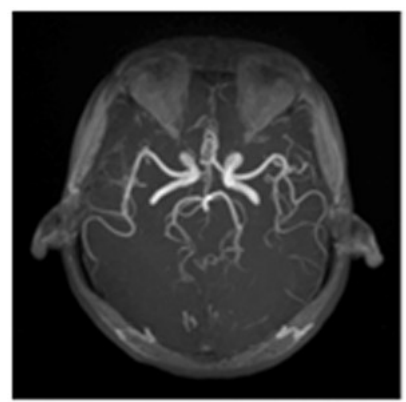

(b)

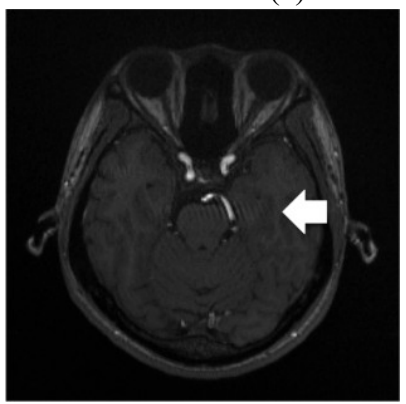

(d)

Figure 6. The MRA images acquired by reverse elliptical centric dual-echo 3D TOF (a) and conventional dualecho 3D TOF (b) acquisitions (c) and (d) are one of the images acquired by reverse elliptical centric dual-echo 3D TOF pulse sequence and conventional dual-echo 3D TOF pulse sequence, respectively.

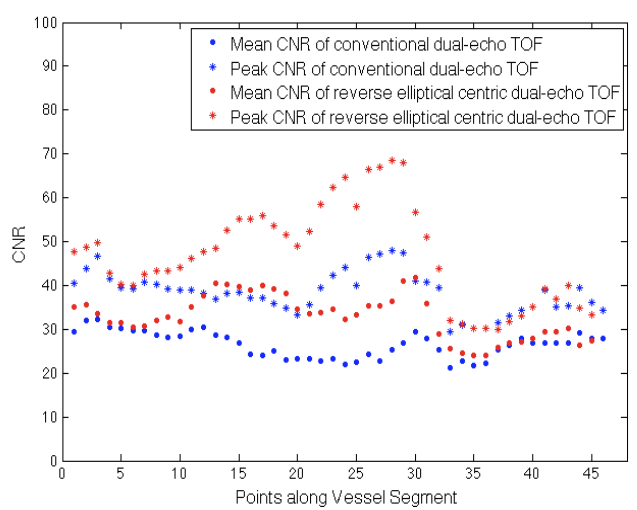

(a)

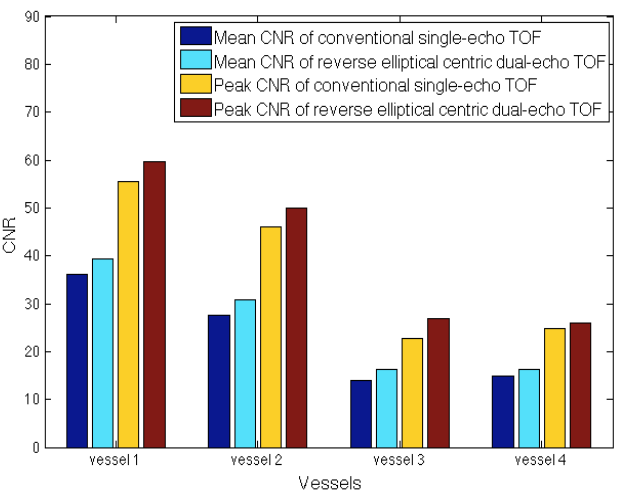

(b)

Figure 7. The mean CNR and peak CNR of the points along the vessel labeled " 1 " in conventional dual-echo and reverse elliptical centric dual-echo 3D TOF acquisition are shown in (a). A histogram shown in (b) is used to graphically summarize and display the mean CNR and peak CNR of four vessels averaged from five volunteers.

Fig. 6 shows the MRA data acquired by the reverse elliptical centric dual-echo 3D TOF (the left column) and the conventional dual-echo 3D TOF (the right colu mn) acquisition, respectively. The top row shows the MIP of MRA, and the bottom row shows the specific slice of the same patient. In the bottom row, the artifacts indicated by the arrow in Fig.6 (d) were reduced much in the reverse elliptical centric dual-echo 3D TOF. The artifacts due to the off-resonance effects were predicted theoretically by PSF analysis ${ }^{15}$. Beyond that, the local vess els CNR of the four vessels were measured 
to compare in Fig.7 (a) and (b). Hence, compared with the conventional dual-echo 3D TOF MRA, the current method not only reduce the scan time by $22 \%$, but also decrease the artifacts and improve the CNR of images.

To further compare the reverse elliptical centric dual-echo 3D TOF and the conventional singleecho 3D TOF pulse sequence, Fig 8.(a) and Fig 8.(b) show the MRA data acquired by reverse elliptical centric dual-echo 3D TOF pulse sequence after averaging and conventional single-echo 3D TOF pulse sequence, res pectively.

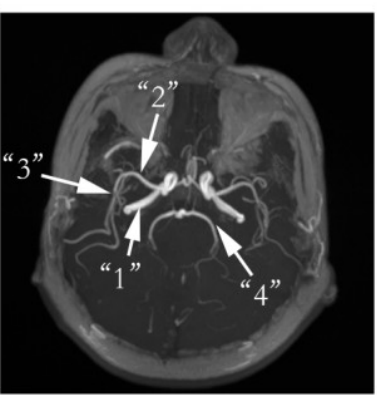

(a)

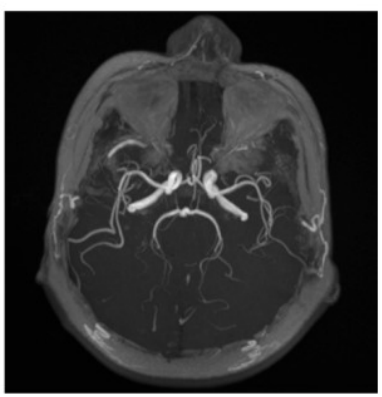

(b)

Figure 8. The MRA images acquired by reverse elliptical centric dual-echo 3D TOF after averaging (a) and conventional single-echo 3D TOF (b) acquisitions.

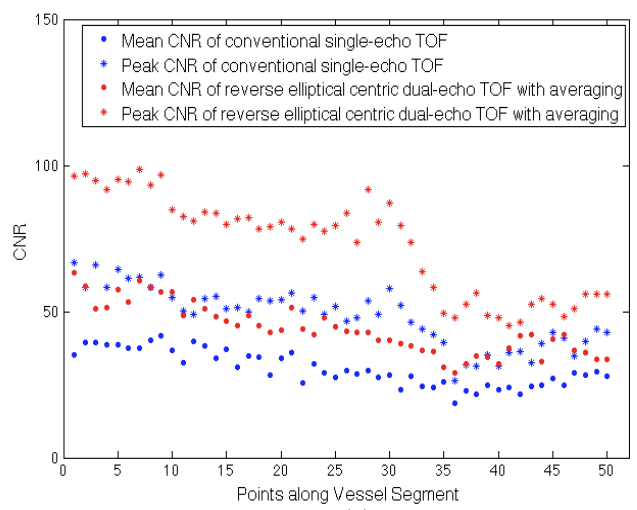

(a)

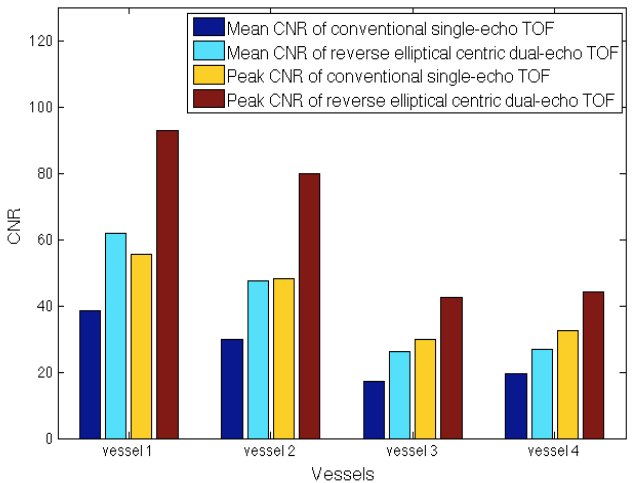

(b)

Figure 9. The mean CNR and peak CNR of the points along the big vessel labeled " 2 " in conventional singleecho and reverse elliptical centric dual-echo 3D TOF with two-average acquisition are shown in (a). A histogram shown in (b) is used to graphically summarize and display the mean CNR and peak CNR of four vessels averaged from five volunteers.

And the CNR measurements were made in the Fig.9. For the big vessels like "1" and "2", the CNRs of reverse elliptical centric dual-echo 3D TOF MRA with averaging are much higher. In addition, the CNRs of s mall vessels such as " 3 " and "4", are also higher.

\section{Discussion}

The applications of MRA always confront two problems. (1) Due to the long scan time, it may bring the motion artifacts and also occupy the time required for other scans, affecting the effective diagnosis of the patient's condition. (2) In vascular MRI, the phase dispersion caused by complex flow might lead to the signal loss, which can be avoided by reducing TE. The developed pulse sequence can resolve the two problems by reducing the scan time to about $39 \%$ of the conventional method and decreasing the effective TE from $4.8 \mathrm{~ms}$ to $4.1 \mathrm{~ms}$. 
Table 1. Mean and peak CNR of the vessels

\begin{tabular}{|c|c|c|c|c|}
\hline & $\begin{array}{c}\text { Conventional } \\
\text { single-echo } \\
\text { TOF }\end{array}$ & $\begin{array}{c}\text { Conventional } \\
\text { dual-echo } \\
\text { TOF }\end{array}$ & $\begin{array}{c}\text { Reverse elliptical centric } \\
\text { dual-echo TOF }\end{array}$ & $\begin{array}{c}\text { Reverse elliptical centric } \\
\text { dual-echo } \\
\text { averaging }\end{array}$ \\
\hline Mean/Peak & $26.29 / 41.47$ & $23.11 / 37.29$ & $25.63 / 40.64$ & $40.56 / 64.85$ \\
\hline
\end{tabular}

TABLE I shows the mean and peak CNR of the MRA of the vessels averaged from the five volunteers acquired by different acquisition methods. Co mpared with the conventional single-echo 3D TOF, the mean and peak CNR of reverse elliptical centric dual-echo 3D TOF MRA are decreas ed by $2.5 \%$ and $2 \%$, respectively. Compared with the conventional dual-echo $3 \mathrm{D}$ TOF, the mean and peak CNR of reverse elliptical centric dual-echo 3D TOF MRA are increased by $10.9 \%$ and $8.9 \%$, respectively. In addition, the comparis on of CNR between the conventional single-echo TOF MRA and the reverse elliptical centric dual-echo TOF MRA with two-average shows that the mean CNR and peak CNR are increased by $54.3 \%$ and $56.4 \%$, respectively. In conclusion, the results reveal the CNR advantage of reverse elliptical centric dual-echo 3D TOF pulse sequence.

\section{Acknowledgment}

The author thanks JinWang, Lele Zhao, Suhang Mao, Xiaoqian Huang, Ke Meng and Tianyi Zeng, for their assistance in the preparation of this paper. This study was greatly supported by the National Natural Science Foundation of China (61372024, 81371518).

\section{References}

1. S.N. Urchuk, D.B. Plewes, JMRI, Mechanisms of flow-induced signal loss in MR angiography, 2(4): 453-462 (1992)

2. H.T.C. Nielsen, G.E. Gold, E.W. Olcott, et al, MRM, Ultra-short echo 2D time-of-flight MR angiography using a half-pulse excitation, 41(3): 591-9 (1999)

3. A.J. Evans, D.B. Richardson, R. Tien, et al. AJNR, Poststenotic signal loss in MR angiography: effects of echo time, flow compensation, and fractional echo, 14(3): 721-9(1993)

4. P. Schmalbrock, C. Yuan, D.W. Chakeres, et al, Radiology, Volume MR angiography: methods to achieve very short echo times, 175(3): 861-5 (1990)

5. D.L. Parker, C. Yuan, D.D. Blatter, MRM, MR angiography by multiple thin slab 3D acquisition, 17(2): 434-51 (1991)

6. W. Fang, Y.P. Du, BMEI, Optimization of 3D time-of-flight MR angiography with shortened echo time and dual-echo acquisition, 53-58 (2013)

7. K. Ying, P. Schmalbrock, B. Clymer. MRM, Echo-Time Reduction for Sub millimeter Resolution Imaging with a 3D Phase Encode Time Reduced Acquisition Method, 33(1): 82-87 (1995)

8. EM. Haacke, RW. Brown, MR. Thomson, R. Venkatesan. Wiley-Liss, Magnetic resonance imaging: physical principles and sequence design (1999)

9. M.A. Bernstein, S.B. Fain, S. J. Riederer, JMRI, Effect of windowing and zero-filled reconstruction of MRI data on spatial resolution and acquisition strategy, 14(3): 270-80 (2001)

10. King, K. Franklin, Elsevier, Handbook of MRI Pulse Sequences, 687 (2004)

11. F. R. Korosec, MRA, Basic Principles of MRI and MR Angiography, 3-38 (2004)

12. T. Doi, T. Okamoto, K. Nakagawa, Japanese Journal of Radiological Technology, The partial volume effect in MRI, 51 (1995) 
13. Yiping P. Du MS ME, D.L. Parker, W.L. Davis, et al, JMRI, Reduction of partial-volume artifacts with zero-filled interpolation in three-dimensional MR angiography, 4(5): 733-741 (1994)

14. Y. Du, D.L. Parker, W.L. Davis, et al, Investigative Radiology, Contrast-to-noise-ratio measurements in three-dimensional magnetic resonance angiography, 28(11): 1004-9 (1993)

15. E. K. Jeong, D.L. Parker, J. S. Tsuruda, et al, MRM, Reduction of flow-related signal loss in flow-compensated 3D TOF MR angiography, using variable echo time (3D TOF-VTE), 48(4): $667-76(2002)$ 